Matteo Martelli

Università di Urbino

\title{
Un imaginaire dévoilé/déphasé Stratégies narratives et mémoire culturelle dans le roman 54 des Wu Ming
}

doi: 10.7358/ling-2014-002-mart

matteo.martelli@uniurb.it

\section{INTRODUCTION}

Depuis quelques années, la critique littéraire a notamment indiqué que l'un des traits communs de la prose narrative italienne récente est une tendance au réalisme qui se réalise sous de nombreuses formes: roman historique, roman noir, récit autobiographique, autofiction, ou encore formes d'enquête et formes littéraires hybrides. Les modalités et les stratégies de représentation de cette tendance ont constitué un thème qui a fortement animé le débat critique, tant du point de vue de la systématisation conceptuelle et stylistique de cette tendance, à savoir l'utilisation et le dépassement d'éléments postmodernes ainsi que de la tradition romanesque, que de celui du rapport entre les éléments de fiction et de non fiction qui ressort des œuvres ${ }^{1}$. De fait, comme l'a souligné Alberto Casadei, les "modalités d'authentification du récit agissent désormais sur des matériaux constamment mélangés, réels-fictifs" ${ }^{2}$ (2007, 54), ce qui pose une question centrale pour définir les approches actuelles de la lecture du réalisme et du rôle qu'y jouent la mémoire collective, les archives, les différents médias et leurs perception et manipulation narratives.

Cette approche trouve un intérêt particulier dans le cadre italien où le lien entre littérature et regard sur le réel s'est fréquemment traduit dans une tendance à enquêter et à élaborer l'histoire récente, en présentant au lecteur des œuvres qui témoignent à la fois d'un engagement des écrivains et d'une

1 Ce débat a été développé dans certaines publications parmi lesquelles Casadei 2007; Donnarumma, Policastro et Taviani 2008; Wu Ming 2009; Spinazzola 2010; Serkowska 2011; Dalmas 2012.

2 Les traductions de l'italien sont de nous. 
confiance renouvelée dans le rôle de la parole écrite. Dans cette perspective, le succès de certains genres narratifs, comme le roman noir, souvent penché sur des aspects politiques et sociaux de la période récente, atteste "la nécessité d'une mémoire alternative de type micro- ou macrocollectif" (Jansen et Khamal 2010, 15), donc des formes de construction et de remise en narration d'un passé ressenti comme incertain ou duquel remettre en question les choix interprétatifs dominants.

S'insérant dans cette aptitude, 54 des Wu Ming 3 , le roman qui est l'objet de notre analyse, nous semble un cas d'étude qui met en évidence deux perspectives ressortant de la littérature récente, l'une d'ordre narratif, l'autre théorique. De fait, parmi les écrivains italiens, le collectif Wu Ming a été l'un des premiers à proposer une réflexion d'ordre conceptuel sur les quelques changements littéraires des dernières décennies, en arrivant à en donner une synthèse, notamment avec le mémorandum New Italian Epic (NIE). Dans ces pages, les Wu Ming ont dressé un portrait d'un ensemble d'œuvres, écrites après 1993, qui partagent des traits stylistiques mais aussi éthiques (Wu Ming 2009, IX) et qui ont marqué un détour par rapport à la tradition littéraire italienne des années 80 et du début des années 90. Prenant en considération des romans de différents genres, le mémorandum, entre autres, postule l'affirmation d'une source épique: à savoir des narrations amples et structurellement complexes, souvent focalisées sur des nœuds de l'imaginaire historique ou sur des éléments aventureux de plus ou moins grande envergure et présentés à partir de telles tonalités émotives que le récit est perçu dans une aura de "mythe, un récit capable de faire naitre autour de lui une collectivité, en refondant, par les pratiques de la narration, un discours d'appartenance culturelle, civile et politique" (Benvenuti 2012, 71).

De cette manière, le mémorandum définit un itinéraire, littéraire et politique (Wu Ming 2003, première partie), qui tend à valoriser le rôle des narrations en tant qu'espace d'élaboration des représentations de la réalité (et aussi des visions et des constructions des réalités). Dans ce cadre, si l'analyse des Wu Ming souligne des liens entre l'affirmation d'une forte reprise de la narration à caractère populaire et les événements historiques tant internationaux que plus spécifiquement italiens des vingt dernières années, il nous semble que, comme l'a indiqué Van den Bossche, la spécificité du NIE naît d'un rapport de tension

3 Wu Ming, le nom que le collectif d'écrivains a choisi pour signer ses œuvres après son premier roman $Q$ (1999) signé Luther Blisset, n'est pas un pseudonyme, ni un moyen de cacher les identités des auteurs, qui d'ailleurs sont connues depuis longtemps. Il s'agit plutôt de la recherche d'une "identité collective et multiple" (Napiórkowska 2011, 220-221) qui veut focaliser l'attention du public sur la dimension extra-individuelle et sociale des récits, en dévaluant la figure et la présence de l'auteur comme unique créateur de l'œuvre (Wu Ming 2003, 8). Sur la formation et l'identité des Wu Ming en tant que collectif, voir De Pascale (2009). 
allégorique qui émerge de ces éléments: plus particulièrement, de l'interaction entre instances allégoriques qui ressortent des œuvres (de leur tonalité épique) et un "allégorisme de deuxième niveau", c'est-à-dire "une nécessité actuelle de liens forts et visibles entre œuvres et contextes sociaux, politiques, culturels" $(2009,74)^{4}$.

C'est selon ce sens que s'éclaircit la description de Wu Ming 2 (Wu Ming $2009,166)$ quand il indique que le travail du collectif est premièrement un travail de transformation. Il s'agit d'une écriture qui, revenant sur ce qui est déjà présent à différents niveaux dans la culture actuelle, en propose, entre autres, une nouvelle expansion de la matière à partir de sources et de témoignages différents (comme les sources orales et les archives), une réinterprétation selon des points de vues inhabituels, une réécriture culturellement engagée (Wu Ming 2009, 165-190) qui ont pour but la mise en place d'histoires alternatives et donc la construction et la réorientation de formes de l'imaginaire (Wu Ming 2009, 164). Si d'une part Raffaele Donnarumma a souligné que, dans cette écriture qui "soustrait du réservoir de la culture de masse en l'hybridant avec lectures d'académie" $(2008,30)$, le modèle qui agit est encore celui des romans d'Umberto Eco, d'autre part, pour Giuliana Benvenuti, cela rejoint

un discours littéraire de type nettement performatif, dans quelques cas connoté par une volonté politiquement orientée, qui se soutient à partir de la persuasion que si on ne peut pas sortir de la société des simulacres avec les armes de l'imaginaire, cependant l'imaginaire contribue à la construction de la réalité. Plus particulièrement, c'est le réemploi de l'imaginaire mainstraem, celui produit par la culture populaire des médias de masse, qui caractérise la recherche de divergences des Wu Ming. (2012, 72)

Si le travail des Wu Ming se penche ainsi sur les imaginaires de la culture populaire, néanmoins il dévoile très ouvertement ces références pour les pervertir, les déphaser et finalement les réécrire selon des orientations différentes. Dans le roman 54, cet exercice tend à la remise en narration d'une mémoire précise, celle de l'après-guerre et son interprétation tant par rapport à la guerre de la libération contre le nazisme et le fascisme (et ses conséquences à l'intérieur de l'Italie), que par rapport à l'image de la naissance du monde contemporain. Cependant, nous envisageons que, à côté de cela, le texte offre une plus ample réflexion qui a pour but une confrontation avec le destinataire. Il s'agit, dans ce cas, d'un roman qui retrace l'imaginaire de l'après-guerre pour postuler une quête d'identité (éthiquement et idéologiquement connotée), tant d'un point de vue personnel (des personnages) que collectif, dont le caractère romanesque renforce une dimension allégorique et anhistorique en dialogue avec l'espace culturel extratextuel. C'est sur les stratégies textuelles de manifestation de ces

4 Sur la tension allégorique voir aussi Casadei (2011,3-21). 
thèmes, finalement, que nous nous pencherons de plus près dans cette étude. Nous fractionnerons notre analyse en deux parties dans lesquelles nous verrons que le texte produit un double mouvement: à partir d'une exhibition forte de l'intrigue, le texte étale son élaboration romanesque pour construire une description culturelle dense de l'après-guerre en arrivant à postuler un discours de type performatif (dont le thème est celui de l'indépendance et de l'affirmation d'une identité autonome et de lutte) projeté hors du texte.

\section{EN SUIVANT DES INTRIGUES}

Nous avons synthétisé jusqu'ici des approches théoriques des Wu Ming, en esquissant très rapidement les traits principaux. Dans ce paragraphe, nous nous pencherons différemment sur un premier niveau d'analyse, en nous concentrant sur la structure du récit à partir de son intrigue. En effet, du point de vue de la construction du roman (mais aussi de l'idée du roman qui ressort du texte), 54 est en premier lieu un dispositif puissant et complexe où la manifestation de l'intrigue est la motrice principale. De manière plus ample, ce qui est mis en scène dans le livre, c'est d'abord l'exubérance et l'abondance du récit: sa capacité d'aborder et de réélaborer différents genres (roman d'espionnage, d'action, d'initiation, de formation, etc.), de développer quelques dizaines des trajectoires narratives plus ou moins indépendantes les unes des autres, de mettre en place un nombre encore plus grand de personnages, tant fictifs qu'ayant réellement vécu.

En l'absence d'un nœud prédominant autour duquel les autres trames se réalisent, le roman se développe à partir d'une série de parcours qui se focalisent sur différents personnages et qui se rattachent de temps en temps donnant forme à une structure dense, stratifiée, qui avance par la juxtaposition et l'alternance de scènes autonomes. Cela favorise une lecture superficielle de l'histoire en tant que divertissement, souvent comique et hyperbolique, tout en proposant une relecture du turning point à partir duquel se sont formés les actuels équilibres historiques et politiques, comme les mettent en lumière les deux brefs résumés journalistiques suivants, destinés au grand public:

[The novel] is a baggy story of post-war Italy at the height of the cold war. This diverting post-modernist entertainment - about narcotics, the cheap potency of Hollywood, the coming of television, the balance of political power, and how the effects knock on down the line - is skilled in teasing fact and fiction. Trieste sits uncomfortably between east and west while in Naples, the deported American gangster Lucky Luciano fixes horse races and attends to his global drug trade. Meanwhile, Cary Grant, idling in semi-retirement in Palm Springs, is approached by British intel- 
ligence to undertake a secret mission to Yugoslavia as part of an incentive to woo Tito, who is interested in a film about his war exploits. (Petit 2005)

54 takes place over that single year, half a century ago. At this crucial turning-point - so the novel claims - Italy's and Europe's hopes of radical change foundered in the face of Hollywood escapism, lifestyle dreams, Cold War intrigues and the might of the US-backed elites. (Tonking 2005)

Ces extraits mettent en évidence que dans le roman se joignent au moins deux instances principales traitées, comme l'a affirmé Wu Ming 1 (Amici 2010, 7-8), par un style principalement comico-grotesque et maintenues ensemble à partir de trois grands axes. Le premier parcours est celui qui se forme à partir du personnage de Robespierre Capponi. Autour de celui-ci s'ouvrent quelques thématiques qui caractérisent la réflexion sur la condition italienne des années 50 que les Wu Ming avaient longuement affrontée dans leur œuvre précédente Asce di guerra. Il s'agit notamment d'un parcours sur la mémoire culturelle qui tend à remettre en narration les traumatismes de la période présents au-delà de l'image de la "pace sociale e politica [cristallizzatasi] nel cinema, mentre tutto ciò che non assecondava quel desiderio veniva rimosso, censurato" (Wu Ming et Ravagli 2005, 33: "paix sociale et politique [cristallisées] surtout par le cinéma, alors que tout ce qui ne secondait ce désir était détourné et censuré"). Le deuxième axe narratif est construit autour de Stefano Zollo, chauffeur de Lucky Luciano, le gangster installé à Naples où il contrôle le trafic d'héroïne. L'axe se présente comme un récit d'action penché sur les connexions globales de l'illégalité, en explorant en même temps un autre aspect du pays: "l'arretratezza dell'Italia" (Wu Ming 2008, 96: "l'arriération de l'Italie"), son "aria di provincia e di sporco appena sciacquato via" (Wu Ming 2008, 157: "air de province et de saleté à peine enlevée"). Enfin, le troisième parcours est celui de Cary Grant, l'acteur hollywoodien qui dans le roman joue le rôle d'agent secret, avant de jouer dans le film d'Hitchcock To catch a thief. En parallèle à l'histoire de Robespierre Capponi, plusieurs thématiques, ancrées sur les fils de la guerre froide et de ses intrigues secrètes entre Hollywood et l'Europe, s'ouvrent autour de Cary Grant.

Ces intrigues sont animées par des mouvements de dispersion et de désorientation du lecteur liés aux éléments de complication de la trame et à sa présentation à partir d'un montage alterné des chapitres et par d'autres mouvements (mineurs mais décisifs) d'organisation et de synthèse. De fait, la fréquence d'apparition de chaque fil narratif se modifie en correspondance avec les points de tension selon une construction presque cinématographique: une intrigue est d'abord diluée et dispersée parmi les autre histoires, alors qu'en arrivant aux moments de forte dramatisation on assiste à un rapprochement des chapitres, quoiqu'ils restent le plus souvent non conséquents, selon une technique de jux- 
taposition très rythmée qui se répète pendant tout le roman. Un autre facteur qui intervient dans les processus de dispersion est la manière de focaliser le récit: c'est ce que dans le mémorandum les Wu Ming ont appelé "lo sguardo obliquo" (2009, 26-32: "le regard oblique"), c'est-à-dire une focalisation mobile, souvent marginale et inattendue, qui tend à "révéler progressivement les contours d'un événement, grâce aux déplacements contigüs de perspective dus à la subjectivité des personnages" (Piga 2011a, 311).

De fait, dès le prologue, le récit présente de façon non connectée les différents nœuds du récit, entrecoupant des chapitres très brefs (de deux à dix pages environ) où le narrateur adopte chaque fois le point de vue du sujet de l'action, tant quand il est un personnage que quand il est un objet. Comme l'a signalé Emanuela Piga, cette technique de focalisation de la matière narrative participe à la construction d'une ambiance historique narrativisée selon différentes microhistoires qui

put $[\ldots]$ the individual, implied in the situated point of view, at the heart of research, in order to reveal a network of connections, situations, transactions, systems of beliefs and group identities. The object of analysis, as a bundle and network of relations, is inserted into a comparative viewpoint, which serves to emphasize its micro-perspective. $(2010,54)$

La dimension de l'intrigue, dans sa propension à la multiplicité et à la génération de points de vue marginaux, représente donc un pivot investi d'un rôle tant narratif qu'herméneutique. Multipliant les trajectoires romanesques, le texte affirme une nécessité de rendre compte du réseau qui définit une prise complexe sur l'histoire, mais aussi le dévoilement de plus près de ses bruissements expulsés de l'image de la période que l'usage politique de la mémoire nous a transmise (Wu Ming et Ravagli 2005, 32). L'explosion des focalisations transversales est dans ce sens reprise par l'explosion géographique des intrigues: le déplacement constant des héros du récit, ne se réalisant presque jamais de façon ordinaire, renforce l'image d'une série de liaisons qui demeurent dans le domaine du secret, du non-dit, ou de l'oublié. C'est dans ces pérégrinations sous mimétisme que se montrent les jonctions formées par "l'histoire officielle" et la possibilité, pour les figures du récit, de croiser des autres personnages et de relier les différentes microhistoires. Comme l'a indiqué Bruno Blanckeman pour des autres romans de l'extrême contemporain, "l'intrigue se décale, se dédouble, se défait. Un jeu sur les proportions romanesques en redouble l'effet: les situations prolifèrent, les circonstances rebondissent, les aventures s'amalgament" $(2000,16)$.

Cependant, cette focalisation multiple et mouvante offre au lecteur un espace de vision chaque fois fortement étroit qui détermine, surtout dans la première partie du livre, une difficulté à encadrer les événements et les per- 
sonnages. Tout cela est à l'avantage de deux conditions de lecture. D'un côté, le lecteur dépaysé s'appuie plus fréquemment sur ces éléments qu'il trouve plus proches de son encyclopédie (Eco 1985, 95ss) et à partir desquels il peut éclaircir la situation: c'est-à-dire sur les scènes d'encadrement historique, sur les personnages non fictifs les plus reconnaissables, sur les citations de la culture populaire (de films en particulier) qui font déjà partie de son bagage culturel. Nous en avons vu un exemple dans le premier des résumés cités où le sommaire de l'histoire renvoie uniquement aux références connues (Lucky Luciano, Cary Grant, Tito).

D'un autre côté, la façon dispersive de procéder facilite l'attention sur des sentences à caractère général qui le plus souvent fournissent une orientation (et interprétation) de la situation politique. Ces synthèses, qui se répètent dans le roman avec un style très informatif, ne sont pas exprimées seulement du point de vue des personnages ou de celui du narrateur, mais aussi à partir de la présence, dans quatre parties du livre, d'une série de titres de journaux d'époque. Il s'agit, à première vue, de parties d'archive, donc de documents que les Wu Ming ont insérés dans le roman pour en donner le climat politique, étant des publications (Il resto del carlino et L'Unità) perçues, au moins par le lecteur italien, dans leur présence non fictive. Cela n'est pas nouveau pour le collectif d'écrivains qui, quelques années avant 54 , avait publié Asce di guerra, livre qui mélangeait récit de fiction, autobiographie de Vitaliano Ravagli et documents sur la lutte partisane et sur l'après-guerre. Mais, si l'utilisation des documents offre au lecteur une "reconstruction indiciaire" (Chimenti 2011, 322) de la période, ces titres, en parallèle à la réécriture des personnages ayant réellement vécu, ont subi des manipulations (en connotant différemment certains mots et en faisant des collages entre différentes parties des titres). Le document devient ainsi moins fiable; en même temps, parce qu'il n'est pas demandé au lecteur de connaître ou de prendre en charge de façon critique la manipulation, il assume dans le texte une capacité de synthèse très puissante. L'espace du connu, l'espace qui dans le roman assume ce qui est immédiatement reconnaissable, peut donc devenir flou selon l'orientation de la transformation des références. De plus, cette orientation peut finalement être plus ou moins manifeste, donc reliée aux mouvements de dispersion ou de synthèse, jusqu'au cas cité où la fidélité est donnée à voir sans qu'elle soit reportée aux contenus.

Dans ce niveau de lecture des intrigues, nous voyons que la réorientation des contenus donnés est l'une des stratégies les plus à l'œuvre. Il est possible de prendre comme exemple l'un des parcours principaux, celui de Gary Grant. Dans la première partie du livre (58 chapitres), les séquences qui regardent directement ou indirectement l'histoire du personnage de Grant sont distribuées de manière non homogène, mais selon quatre sections d'ampleur différente qui contiennent 17 chapitres. Avant même que le personnage entre en scène, il est 
aussi évoqué en tant que star hollywoodienne: Lucky Luciano imagine un film sur sa vie avec Grant comme protagoniste, alors que Robespierre Capponi, en regardant une photo dans sa chambre, essaie d'imiter (de devenir) l'acteur (Wu Ming 2008, 30 et 47-47). Quand finalement le personnage entre en scène, c'est encore sous cette forme que nous le voyons dans la première section: Cary Grant, dans sa maison de Palms Spring, qui hausse "il sopracciglio sinistro in un'espressione di sorpresa già vista al cinema" (Wu Ming 2008, 93: "le sourcil gauche dans une expression de surprise déjà vue au cinéma") face aux agents britanniques qui l'engagent dans la mission européenne (le film sur Tito). Il s'agit donc d'une présentation qui se focalise sur l'homme hollywoodien, "il perfetto prototipo di Homo Atlanticus" (Wu Ming 2008, 60: "le parfait prototype d'Homme Atlantique"), en reportant dans le texte une figure très connue: l'acteur caractérisé par son élégance et sa perfection. C'est la facilitation offerte au lecteur qui peut immédiatement encadrer le personnage. En même temps, le texte propose une seconde image, celle d'Archie Leach (le vrai nom de l'acteur) plus ambigüe parce que moins connue et présentée en parallèle avant d'être superposée à Grant, dans la figure du double.

Cary si radeva con la destra, apprezzando ogni istante di quella liturgia mattutina [...]. Archi, che era mancino, si sfiorò le guance col palmo della sinistra chiuso a conchiglia. Due giorni senza radersi e senza alcuna voglia di farlo. [...] Ogni settimana le manicure $[\ldots]$ ammorbidivano le mani di Cary, mani che ogni donna dell'universo avrebbe voluto sotto la sottana o intente a sbottonarle la blusa, ma il tessuto calloso riprendeva a crescere, souvenir della vita precedente, il passato di Archibald Alexander Leach. (Wu Ming 2008, 58)

Cary se rasait avec la main droite, en appréciant chaque instant de cette liturgie du matin [...]. Archi, qui était gaucher, toucha ses joues avec la paume de la main gauche fermée comme un coquillage. Deux jours sans se raser et sans aucune envie de le faire. $[\ldots]$ Chaque semaine les manucures $[\ldots .$.$] assouplissaient les mains de Cary,$ mains que chaque femme de l'univers aurait voulu sous son jupon ou en train de lui déboutonner le chemisier; mais les durillons recommençaient à se manifester, souvenir de la vie précédente, le passé d'Archibald Alexander Leach.

La juxtaposition des "deux hommes", le prolétaire britannique et l'acteur réussi, ne se résout pas dans une tension psychologique ayant pour but de faire connaitre les profondeurs du personnage. L'introspection qui émerge se relie à la dimension de la réorientation des contenus connus selon l'effet identifié. De fait, le lecteur est invité à suivre d'abord ce qui est immédiatement reconnaissable, qui est dans la scène, en plus du personnage de Grant, la longue explication que les agents britanniques font, de façon très informative, de la situation géopolitique mondiale (Wu Ming 2008, 103). Ce résumé des straté- 
gies et des configurations de la guerre froide est cependant repris et modifié par des interventions intermittentes: c'est le double de Grant, Archie Leach, qui offre une lecture alternative de ce qui advient dans le cadre occidental, à partir de son "disgusto [...] per le scorribande di Joe McCarhty e dei suoi tirapiedi" (Wu Ming 2008, 61-62: "dégoût [...] pour les incursions de Joe McCarhty et de ses larbins"), donc pour la "chasse aux sorcières" que la commission présidée par le sénateur américain avait menée contre de nombreux intellectuels et personnes du spectacle. Il s'agit d'une tension stratégique qui multiplie et réoriente les interprétations que le lecteur a déjà lues, et qui se poursuit dans les autres chapitres de l'histoire de Cary Grant, dans lesquelles l'acteur n'est pas sujet de l'action. Ces passages reprennent l'histoire du personnage et de la mission d'un autre point de vue (celui des agents soviétiques du KGB) formant, encore une fois, une réorganisation du contenu. L'intrigue revient sur elle-même, se réécrit et se transforme, se montrant clairement en tant que jeu stratégique: un dispositif qui procède selon une "thick description" (Geertz 1998, 12-25), une reconstruction des réseaux du savoir où les éléments décrits font sens globalement, assumant leur signifié l'un en fonction de l'autre (Lorusso 2010, 124). Enfin, ce qui se montre à partir de la structure de l'intrigue est une figure herméneutique, la figure du réseau.

L'aventure romanesque est donc exhibée en tant qu'entrelacement qui "capte chacune des autres formes du savoir" (Santoro 2007, 5) avec lesquels elle instaure une relation critique. C'est notamment le cas du niveau des citations, qui tendent à encadrer le texte, comme il advient avec le roman que Grant lit pendant son voyage: Casino Royale de Ian Fleming, la première œuvre de la saga de James Bond sorti en 1953. Ce roman est commenté à plusieurs reprises par le personnage, qui exprime un jugement négatif (mais pour le lecteur plein d'ironie):

A proposito di storie, ho letto un libro ridicolo e disgustoso, scritto da un certo Fleming. Il protagonista è un agente M16 di nome "James Bond". Brevi cenni. Sconclusionato, invero. Ecco un libro da cui non trarranno mai un film! Risate. (Wu Ming 2008, 388)

À propos d'histoires, je lus un livre ridicule et dégoûtant, écrit par un certain Fleming. Le protagoniste est un agent du M16 nommé "James Bond". Brefs aperçus. Incohérent, hein! Voilà un livre duquel ne jamais faire un film! Éclats de rires.

Le clin d'œil au lecteur est la boutade finale d'une série d'informations que le texte donne sur le roman de Fleming, mettant en scène un parallélisme entre deux livres ancrés dans la même période. La citation, dans sa redondance, n'est pourtant pas seulement un jeu avec un lecteur plus ou moins averti. Tout ce qui est récupéré, rappelé, modifié, ouvre dans le roman une instance de l'ordre 
de la traduction-transformation entre source et texte d'arrivée. Ainsi, dans les commentaires au livre de Fleming, l'une des références des Wu Ming est en toute probabilité Umberto Eco, qui dans son essai "James Bond: une combinatoire narrative" avait notamment remarqué que les romans de Fleming adoptent une rhétorique qui

fournit l'évasion en contant non ce qui est ignoré, mais du déjà connu. [...] Dans les romans de Fleming, on célèbre donc de façon exemplaire cet élément de jeu escompté et de redondance absolue qui caractérise les instruments d'évasion fonctionnant dans le domaine des communications de masse. (1966, 90-91)

Cette annotation d'Umberto Eco nous montre que le roman des Wu Ming partage des éléments avec le livre de Fleming: l'utilisation et la mise en lumière du déjà connu et de la redondance dans la progression de l'intrigue. Cependant, le parallélisme exprimé dans 54 engendre une confrontation polémique avec le livre de James Bond, c'est-à-dire avec une structure narrative traditionnelle qui est reprise et approfondie dans l'écriture de transformation adoptée par les Wu Ming. Si l'intrigue de Fleming tend à se présenter comme immédiatement perceptible, en manifestant ce que le lecteur peut connaître, et procède par la mise en scène d'oppositions élémentaires (Eco 1966, 90-92), le roman 54 dissémine et disperse dans sa narration les détails d'un imaginaire consolidé en les joignant dans de nouvelles séquences de sens à partir d'une perspective multifocale. D'un côté, le roman se met ainsi en relation avec des structures que lui-même adopte et reprend et à partir desquelles il pose la centralité de la dimension de la narration; d'un autre côté il établit une confrontation culturelle avec les textes qui ont marqué une description, fictionnelle mais très connue, de la période, qu'il s'agisse de l'approche manichéiste de Fleming (Eco 1966, 91) ou de celle du scénariste italien du Néoréalisme Cesare Zavattini auquel est contestée, dans un long commentaire de l'un des ces films, Siamo donne (1953), une fausse représentation de la condition de la pauvreté et des désirs du monde populaire italien (Wu Ming 2008, 133-136). Cette façon d'accueillir dans l'écriture une pluralité de matériaux, et leurs façons de rentrer en dialogue dans le roman, doivent être reconduites au rôle du pivot de la tension de l'intrigue. Comme l'a dit Simonetti, c'est le "manque de linéarité [et la] structuration discontinue" $(2012,116)$ du romanesque qui forment un effet de réalité pluralisée où interviennent encore des techniques de la littérature de genre inspirées de "la communication audiovisuelle de masse comme un modèle d'énergie narrative, atlas linguistique et schéma rythmique" (118). 


\section{UN RÉGIME HITCHCOCKIEN}

La forme des intrigues, que nous avons vue à partir du cas de Cary Grant, s'appuie donc sur une relation constante entre des éléments connus et des focalisations alternatives, qui prévoient une révision des contenus textuels et souvent leur redoublement qui comporte des variations. Le procès est encouragé par la manière de donner les informations de la part du narrateur qui utilise amplement une forme d'attente spécifique, celle des incomplétudes stratégiques. Comme l'a notamment indiqué Raphaël Baroni, "les romans de grande consommation démontrent à l'évidence que les stratégies narratives du suspense et de la curiosité sont des moyens extrêmement efficaces pour attirer et satisfaire les demandes du public, comme en témoignent les tirages importants des romans noirs, policiers, d'espionnage ou d'action" (2004, 31). Ces incomplétudes ne sont pourtant pas envisagées seulement pour une question de succès, mais elles jouent un rôle herméneutique et définissent un type d'investissement du lecteur par rapport aux sujets du récit. De cette façon, Baroni fournit une définition opérationnelle du suspense:

Le suspense serait quant à lui créé par un événement initial ayant la potentialité de conduire à un résultat important (bon ou mauvais) pour un ou plusieurs des personnages principaux [...]. La tension de la lecture est alors orientée vers les événements à venir et vers le non-encore-dit du texte qui est ici déterminant. $(2002,117)$

Cette orientation de la lecture peut se former aussi à partir d'une série d'informations dont l'interprétation est difficile ou lacunaire. Le narrateur, qui omet volontairement une partie de la référence de son discours, qui rend visible un manque, qui anticipe des éléments incongrus ou qui, de façon équivoque, met à l'épreuve le régime d'attente du lecteur, encourageant (et orientant) la construction d'hypothèses à partir desquelles résoudre les incertitudes.

En suivant cette interprétation du suspense, nous pouvons distinguer deux régimes différents présents dans le roman des Wu Ming. Le premier se réfère à la condition de suspense en tant que stratégie d'animation du texte, le second à une stratégie d'élaboration de la matière représentée.

L'animation du roman suit les lignes des intrigues, en respectant d'ailleurs des règles romanesques qui encadrent 54 comme un roman d'espionnage et d'aventure. Il s'agit donc d'une technique que les Wu Ming adoptent dans le déroulement de leur texte, intervenant sur "la dramatisation du matériel narratif $[\ldots]$ ou encore [sur] la présentation la plus intense possible des situations dramatiques" (Truffaut 1966,13) qui rappellent une manière de procéder hitchcockienne. Effectivement, le réalisateur britannique est tant l'un des personnages de l'histoire que l'une des ultérieures références dans le jeu entre le connu et l'inhabituel. Rappelons que dans le roman Hitchcock est en train de préparer 
le tournage de son film To catch a thief (qui est défini ironiquement par Grant "una bella storia, anche se avrei preferito più suspence" [Wu Ming 2008, 148: "une belle histoire, quoique j'aurais préféré plus de suspense"]); que le voyage en incognito de Cary Grant pour le service secret anglais est fait sous le nom de "George Kaplan", qui sera, peu d'années après, l'inexistant agent du FBI pour lequel est pris le personnage de Grant, Roger Thornhill, dans North by Northwest (1959) de Hitchcock; ou encore que le téléviseur (vide à l'intérieur) qui parcourt tout le texte est nommé McGuffin, rappelant l'objet-prétexte notamment utilisé par le réalisateur dans ses films (Truffaut 1966, 100-102).

Dans le cadre des références au "maître du suspense", il faut rappeler une autre notation de Baroni, pour lequel l'incomplétude narrative du suspense agit en favorisant l'identification aux personnages de l'histoire selon un "investissement affectif" et aussi renforce "l'illusion mimétique, [en affirmant] cette impression fausse que les mots seraient transparents et qu'ils donneraient accès à un univers 'réel', qui ne serait pas que de papier" (2004, 36). De manière semblable, on retrouve cette illusion chez Hitchcock:

Hitchcock dit souvent qu'il se moque de la vraisemblance, mais en fait il est rarement invraisemblable. En vérité, il organise ses intrigues à partir d'une énorme coïncidence qui lui fournit la situation forte dont il a besoin. Ensuite, son travail consiste à nourrir le drame, à le nouer de plus en plus serré en lui donnant le maximum d'intensité et de plausibilité avant de le dénouer très rapidement après un paroxysme. (Truffaut 1966, 13)

De la même façon, les Wu Ming ne s'empêchent pas d'insérer dans leur histoire les faits les plus arbitraires; et l'on pourrait dire que tout le roman du point de vue de l'intrigue n'est qu'une construction extrêmement capricieuse. Cela est possible parce que ce que les auteurs recherchent est la plausibilité de l'histoire dans son déroulement, et non sa plausibilité en tant que fait historique: "Tito e Cary Grant conversavano amabilmente. Si può pensare a una scena più surreale? Niente ha senso, tranne il fatto che sono qui e mi sento bene" 5 (Wu Ming

5 Le même concept est repris à la fin du livre: "Pero in questo posto c'è una regola non scritta: tutti quelli che passano di qui hanno una storia da raccontare. A volte è vera, altre volte pura fantasia. Non fa molta differenza, se è una buona storia" (Wu Ming 2008, 655: "Ici, il existe une règle non écrite: tous ceux qui viennent ont une histoire à raconter. Parfois elle est vraie, d'autres fois elle est une invention de la fantaisie. Cela ne fait aucune différence si l'histoire est un bon récit"). Comme l'a indiqué Santoro: "Alors, les moyens comme le suspense et l'effet de distanciation, qui font partie de la littérature de genre, sont aussi fonctionnels par rapport à la tentative de répondre au mieux à la demande d'orientation cognitive que le lecteur pose à l'écrivain, désormais vu comme porteur d'une mathesis plutôt que d'une mimesis. C'est-àdire, comme celui qui, mélangeant constamment différents niveaux, de l'hyper-fictionnel au document, fait ressortir la contradiction entre un réel filtré par les écrans des télévisions et une construction littéraire qui tend à devenir exclusivement parodique et combinatoire" $(2007,11)$. 
2008, 310: "Tito et Cary Grant bavardaient aimablement. Est-ce qu'on peut penser une scène plus surréelle? Rien n'a de sens, sauf le fait que je suis ici et que je me sens bien"). La combinaison arbitraire des événements du récit est donc basée sur la construction d'une attente qui lie le lecteur à ce déroulement particulier.

La valeur du suspense hitchcockien est donc réélaborée dans la dynamique de l'intrigue, qui fournit au destinataire des anticipations à partir desquelles focaliser l'attention et orienter les hypothèses de lecture. Nous retrouvons cette technique dans l'aventure de Stefano Zollo qui a pour objectif de voler de la drogue à Lucky Luciano, la revendre et s'en aller en Amérique. Son parcours, le plus fragmenté, se conclut avec la tentative de vente pendant laquelle il est assassiné. Dans la description de l'histoire, le roman d'abord postule un régime de curiosité: la drogue, accumulée avec péril et difficulté, se trouve à l'intérieur du téléviseur McGuffin qui, par un long moment, reste hors du contrôle de Zollo obligeant ce dernier à la récupérer de manière aventureuse. Une fois l'héroïne retrouvée et l'accord de vente établi, un chapitre d'une demi-page interrompt ce récit. Ce sont trois brefs paragraphes, en discours indirect libre focalisé sur Lucky Luciano: Zollo a été découvert, le boss cherche sa vengeance.

Le lecteur participe évidemment de façon toute différente aux chapitres qui suivent la révélation sur Zollo, en imaginant déjà que la livraison sera un piège. Mais pour le narrateur, le suspense n'a pas de valeur en soi: il sert à créer un régime de participation et d'attention émotif et intense pour parvenir à un déphasage. Ainsi, cette dramatisation correspond finalement aux chapitres où Ettore, un ex-partisan qui est avec Zollo, divulgue son histoire et les motivations de sa chute dans l'illégalité. Nous assistons ainsi à la mise en narration d'un thème fort du livre, celui du changement manqué de l'Italie après la guerre:

L'Italia è uno stivale, noi abbiamo provato di lustrarlo, ma il posto di uno stivale resta sempre il fango. Prima, almeno, le cose erano chiare: lo sapevano tutti che se non avevi la tessera non potevi lavorare e prendevi anche le botte. Adesso la fanno più sporca, perché c'è la democrazia. La legge non è uguale per tutti. Se tu c'hai degli amici, [...] diventi ricco e nessuno ti viene a dir niente. Altrimenti, ciccia. [...] E intanto i delinquenti veri fanno i milioni. (Wu Ming 2008, 586)

L'Italie est une botte; nous avons essayé de la cirer, mais la place d'une botte reste toujours la boue. Avant, au moins, les choses étaient claires: tous savaient que si on n'était pas inscrit au parti fasciste, on ne pouvait pas travailler et on était battu. Maintenaient, ils font des choses plus crasseuses, parce qu'on est en démocratie. La loi n'est pas égale pour tous. Si tu connais les justes personnes [...] tu deviens riche et personne ne te dérange. Sinon, rien du tout. [...] Et entre-temps les vrais délinquants se font des millions. 
Comme l'a souligné Salsi (2006), le personnage d'Ettore a une source intertextuelle explicite dans le protagoniste de La paga del sabato de Beppe Fenoglio, figure avec laquelle il partage, outre le nom, une même souffrance existentielle par rapport à la période d'après la Résistance: l'inadaptation aux conditions de la vie ordinaire, l'illégalité, la recherche de l'émancipation personnelle destinée à l'échec. Si, dans 54, cette souffrance est d'abord liée à la problématique de l'après-guerre comme "ritorno dell'Italia a se stessa, alla storia gattopardesca di sempre" (Wu Ming 2005, 256: "retour de l'Italie à elle-même, à l'histoire guépardesque de toujours"), le point de vue développé par le personnage en souligne un aspect particulier: celui de la continuation de la lutte, d'ailleurs celle partisane, mais qui ouvre le thème sur de plus amples motivations pour une résistance généralisée ou pour des formes autonomes, différentes, de résistance individuelle à ce qui est ressenti comme injustice sociale, comme l'indique clairement un autre personnage d'ex-partisan.

La nostra resistenza non è finita quando siamo scesi dalle montagne, continua anche adesso. E se non ci fossimo noi, se ce ne fossimo andati tutti come hai fatto tu, a quest'ora questo paese chissà cosa sarebbe. (Wu Ming 2008, 614)

Notre résistance n'a pas été terminée quand nous sommes descendus des montagnes; elle continue encore ces jours-ci. Et si nous n'étions pas là, si nous étions allés ailleurs comme tu l'as fait, aujourd'hui qui sait ce que serait ce pays.

Ce qui s'éclaircit pour le lecteur, parmi ces confessions dialogiques à la fin du roman, se réfère à l'autre niveau d'incomplétude utilisée dans le texte. Cette forme d'incomplétude a pour caractère de remettre en question des connaissances du lecteur, tout en jouant sur un manque interprétatif qui s'active au début du récit.

En effet, dans un roman qui traverse une année déterminée de la décennie des années 50, c'est d'abord l'encadrement historique qui est mis en lumière: dès la quatrième de couverture, le lecteur se trouve plongé dans une époque qui "non è dopoguerra, è solo un'altra guerra. Ed è un mondo che sta nascendo" (Wu Ming 2008, "n'est pas l'après-guerre, mais seulement une autre guerre. Et c'est un monde qui est en train de naître"). Cette affirmation, qui est reprise, avec peu de variations, au début du roman, demeure ambigüe tout au long du texte, pouvant se référer à la condition de la guerre froide, à la continuation de la guerre partisane sous d'autres formes, ou encore à d'autres variations sémantiques. Les extraits dialogiques reportés répondent à cette ambigüité en l'encadrant dans un contexte social et historique déterminés, celui du mythe de la "Résistance trahie". Néanmoins, une réponse de niveau différent est plus amplement suggérée et représentée dans tout le livre à partir des aventures identitaires des personnages. 
Nous assistons encore une fois à un redoublement stratégique de la situation narrative. De fait, toutes les figures principales essaient d'accomplir un parcours unique et de conquérir le même objet de valeur: c'est-à-dire l'indépendance, l'autonomie par rapport à la société et à la culture de référence, ou encore l'autodétermination de soi. Dès le début du roman, la construction des personnages offre des caractéristiques communes, qui produisent un régime de répétition pressant. Ils partagent ainsi une même instabilité identitaire qui concerne le nom. Ils en ont plusieurs, mais surtout ils arrivent à définir le "moi" seulement en changeant de nom: d'Archie Leach à Cary Grant, de Jorip Borz à Tito, de Robespierre à Pierre (Capponi), de Stefano Zollo à Steve Cemento, de Salvatore Laucania à Charles Luky Luciano, etc. Cette différenciation entre l'identité qui confine le personnage dans l'ambiance de naissance (ou dans le climat culturel de provenance) et celle que lui-même a décidé de poursuivre et de développer en changeant de nom définit les processus de l'autodétermination individuelle qui est mise en contraposition avec le processus historique perçu comme quelque chose dépassant constamment l'individu. L'identité finale est donc une identité qu'il faut conquérir à partir d'une révision du contexte et d'un processus de critique des savoirs dominants. Cette lutte, personnelle et civile, est néanmoins renvoyée hors du livre, ici encore selon des suggestions qui utilisent des imaginaires bien présents dans la culture contemporaine. Effectivement, si nous regardons les protagonistes du roman, ils n'arrivent pas à se réaliser complètement dans leur lutte: Steve Cemento est assassiné pendant la vente de l'héroïne; Robespierre, qui est recherché par la loi et qui s'enfuira au Mexique, le pays des "profughi politici di mezzo mondo" (Wu Ming 2008, 659: "réfugiés politiques de la moitié du monde"), parle de "rivoluzione mancata" (655: "révolution manquée"). Quant à Cary Grant, il a trouvé la manière de dépasser "l'inverno del suo scontento" (Wu Ming 2008, 635: "l'hiver de son mécontentement") dans l'usage de l'acide lysergique, "sostanza atta a 'schiudere lo scrigno dell' $E s$ '" (631: "substance apte à 'ouvrir le coffre de l'inconscient'”).

Le point de vue suggéré, en laissant de côté le personnage hollywoodien de Grant, est donc celui "des vaincus et des oubliés [qui] ne peut jamais être acquis définitivement, parce que l'oubli est une force dynamique, qui continue à avancer" (Amici 2010, 7). Ainsi le texte, mettant en lumière une répétition constante des projets identitaires des personnages, fournit une valorisation positive de leurs tentatives au-delà des aboutissements obtenus. Ce qui est remis en narration n'est donc pas une lutte de libération spécifique, mais une tension à la lutte, comme l'explicite la fin du roman quand Robespierre Capponi en exil rencontre un avocat qui veut libérer son île de la dictature, à savoir Fidel Castro, défini dans le texte fou, mais de la même folie que Don Quijote (Wu Ming 2008, 659).

L'histoire se conclut donc sur une perspective qui, dans l'année 1954, doit 
encore se réaliser, soulignant que la tension de l'intrigue ne tombe pas à la dernière page. L'orientation du texte donnée par le suspense tend finalement à un aboutissement de type performatif: la fin est une anticipation ultérieure dont la valeur ne demeure pas dans le destin de l'île de Cuba, mais dans la construction d'une quête de libération et d'autodétermination (personnelle et/ou collective) qui perdure au-delà de l'espace roman. De cette façon, l'aventure du personnage ne s'achève pas tout à fait: Robespierre, comme d'autres figures du roman, reste face à une situation initiale, avec "una strana sensazione" ("une étrange sensation"), "quella che la vita, come la Storia, non avrebbe smesso di riservare sorprese” (Wu Ming 2008, 659: "celle que la vie, comme l'Histoire, n'aurait pas terminé de garder des surprises”). Ainsi, si l'année choisie pour le roman est ressentie comme le moment où "un monde est en train de naître", la fin du texte perdure sur l'idée du commencement, qui, à un niveau narratif, devient une manière d'intervenir et de s'engager dans une reformulation d'expériences culturelles en se positionnant toujours "à l'origine de l'événement" à partir duquel construire une "narration capable de générer [un] événement collectif” (Piga 2011b, 8) qui n'est pas décrit, mais suggéré, et qui demeure, peut-être, hors du texte.

\section{BIBLIOGRAPHIE}

Amici, Marco. 2010. "Fra narrazioni di trasformazione storica ed etica del mito. Intervista a Wu Ming 1." La libellula 2 (2): 3-14. http://www.lalibellulaitalianistica.it/ blog/wp-content/uploads/2011/02/LaLibellulan2.pdf (3/05/2014)

Baroni, Raphaël. 2004. "La valeur littéraire du suspense." A contrario 1 (2): 29-43.

Baroni, Raphaël. 2002. "Incomplétudes stratégiques du discours littéraire et tension dramatique." Littérature 127: 105-27.

Benvenuti, Giuliana. 2012. Il romanzo neostorico italiano. Storia, memoria, narrazione. Roma: Carocci editore.

Blanckeman, Bruno. 2000. Les récits indécidables: Jean Echenoz, Hervé Gubert, Pascal Quignard. Villeneuve d'Ascq (Nord): Presses Universitaires du Septentrion.

Casadei, Alberto. 2007. Stile e tradizione del romanzo italiano contemporaneo. Bologna: Il Mulino.

Casadei, Alberto. 2011. "Realismo e allegoria nella narrativa italiana contemporanea." In Serkowska ed. 2011, 3-21.

Chimenti, Dimitri. 2011. "La vita postuma delle parole. Note su un uso narrativo dell'archivio in Asce di guerra di Wu Ming." In Serkowska ed. 2011,321-34.

Dalmas, Davide. 2012. "Postmoderno, nuova epica, ritorno alla realtà. Questioni e problemi del romanzo italiano contemporaneo." CoSMo. Comparative Studies 
in Modernism 1: 121-7. http://www.ojs.unito.it/index.php/COSMO/article/ view/160/219.

De Pascale, Gaia. 2009. Wu Ming. Non soltanto una band di scrittori. Genova: Il Melangolo.

Donnarumma, Raffaele. 2008. "Nuovi realismi e persistenze postmoderne: narratori italiani d'oggi." Allegoria 57: 26-54.

Donnarumma, Raffaele, Gilda Policastro et Giovanni Taviani, eds. 2008. "Ritorno alla realtà? Narrativa e cinema." Allegoria 57: 7-93.

Eco, Umberto. 1966. "James Bond: une combinatoire narrative." Communications 8: 77-93.

Eco, Umberto. 1985. Lector in fabula. Traduction par Myriam Bouzaher. Paris: Grasset.

Geertz, Clifford. 1998. Interpretazioni di culture. Trad. Eleonora Bona. Bologna: Il Mulino.

Jansen, Monica et Yasmina Khamal. 2010. "Introduzione. Quale memoria per il noir italiano?" In Memoria in Noir. Un'indagine pluridisciplinare, ed. Monica Jansen e Yasmina Khamal, 9-17. Bruxelles: Peter Lang.

Lorusso, Anna Maria. 2010. Semiotica della cultura. Roma-Bari: Editori Laterza.

Napiórkowska, Izabela. 2011. "Da Luther Blisset a Wu Ming: la poetica della letteratura rivoluzionata.” In Serkowska ed. 2011, 217-31.

Petit, Chris. 2005. "The concept of Cary." The Observer, 21 May. http:// www.wumingfoundation.com/italiano/54/observer_54.htm. (2/10/2013).

Piga, Emanuela. 2010. "Metahistory, microhistories and mythopoeia in Wu Ming." Journal of Romance Studies 10 (1): 51-67.

Piga, Emanuela. 2011a. "A più voci. Testimonianze e narrazione nell'opera di Alessandro Portelli e dei Wu Ming.” In Serkowska ed. 2011, 305-19.

Piga, Emanuela. 2011b. "Memoria, trauma, mitopoiesi. Lawrence d'Arabia e Stella al mattino di Wu Ming 4." Between I (2): 1-13. doi: 10.13125/2039-6597/273.

Salsi, Luigi. 2006. "Quête identitaire et lecture historique. Luther Blisset, $Q$, et Wu Ming, 54." Cahiers d'études romanes 15: 71-124. http://etudesromanes.revues. org/1399.

Santoro, Vito. 2007. "Narrare la post-realtà: la sfida del romanzo contemporaneo." Incroci VIII (16): 136-54. http://vsantoro.files.wordpress.com/ 2008/12/vitoper-incroci-4.pdf: 1-19. (5/07/2013)

Serkowska, Hanna, ed. 2011. Finzione cronaca realtà. Scambi, intrecci e prospettive nella narrativa italiana contemporanea. Massa: Transeuropa.

Simonetti, Gianluigi. 2012. "Il realismo dell'irrealtà. Attraversare il postmoderno." CoSMo. Comparative Studies in Modernism 1: 113-20.

Spinazzola, Vittorio, ed. 2010. Tirature 2010: Il New Italian Realism. Milano: Il Saggiatore. 
Tonking, Boyd. 2005. "A Week in Books: An ingenious comedy-thriller, packed with clever gags." The Independent, 24 June. http://www.independent.co.uk/artsentertainment/books/features/a-week-in-books-an-ingenious-comedythrillerpacked-with-clever-gags-294355.html. (02/10/2013).

Truffaut, François. 1966. Le cinéma selon Hitchcock. Paris: Robert Laffont.

Van den Bossche, Bart. 2009. "Epic \& Ethics. Il NIE e le responsabilità della letteratura." La libellula 1 (1): 68-76.

Wu Ming. 2009. New Italian Epic. Letteratura, sguardo obliquo, ritorno al futuro. Torino: Einaudi.

Wu Ming. 2008. 54. Torino: Einaudi.

Wu Ming. 2003. Giap! Torino: Einaudi.

Wu Ming et Vitaliano Ravagli. 2005. Asce di guerra. Torino: Einaudi.

\begin{abstract}
This essay analyses the narrative strategies through which cultural memory is portrayed in 54, a novel in which Wu Ming (a collective of four Italian fiction writers) suggests a literary mapping of the Fifties. This is obtained by a recovery of a shared imaginary tradition which is close to the reader, mixing fictional and historical characters, films, books, different genres of popular literature. Nevertheless, the rewriting of these imaginary traditions involves their displacement, a presentation which is constantly created from an unusual point of view. Thus, shifting the image of what is already known, the text inquires into several modalities of a questioning about cultural memory and its redefinition in relation to contemporary culture. By focusing on these elements, this study proposes an analysis which starts from the construction of the characters, the role of suspense and the adoption of multiple traditions of novel writing.
\end{abstract}

